\title{
Intellectually gifted and nongifted children's inference from partial knowledge
}

\author{
H. LEE SWANSON \\ University of Northern Colorado, Greeley, Colorado \\ SUSAN KONTOS \\ Iowa State University, Ames, Iowa \\ and \\ CONNELL G. FRAZER \\ West Virginia University, Morgantown, West Virginia
}

(Bill R. Brown, Sponsor)

\begin{abstract}
The present study explored the notion that children of different intellectual abilities vary in their metajudgment when they have partial knowledge. Gifted and average childen rated the likelihood that a protagonist who had knowledge or lack of knowledge about a category of items would remember a particular item of high or low salience. The protagonist was further portrayed as an expert or novice who had previously remembered a large or small set of items. The results show that average children are more likely than gifted children to rate the lack-of-knowledge protagonist as remembering an item. This was especially true when the protagonist was portrayed as an expert who previously had remembered a large number set, and the item to be remembered was highly salient. In contrast, gifted children are less sensitive than average children to the extremity factors of expertise, large set size, and high item salience.
\end{abstract}

The difference between the reasoning of an intellectually gifted child and that of an average child may be not so much a matter of what the gifted child knows as a matter of what the children reason from what they do not know. For example, all children must reason from incomplete or partial knowledge. They must make intelligent inferences about events in life without all the relevant facts. One way gifted children may manage to make decisions in spite of incomplete knowledge is that they take advantage of their superior metaknowledge about the mind (Sternberg, 1982; Sternberg \& Davidson, 1983). That is, they make self-observations on patterns of their knowledge as sources of information.

To test this assumption, intellectually gifted and nongifted children were individually read passages that led them to judge the memory knowledge attributed to a protagonist who was described as having either previous knowledge or lack of knowledge about a particular category of items. The protagonist was further portrayed as an expert or novice who had previously remembered a large or small set of items. After the scenarios had been read, children were asked to rate the likelihood that the protagonist would remember an item. It was reasoned that the scenarios approximated the real world because children must consider multiple pieces (factors) of informa-

The first author's mailing address is: Educational Psychology, University of Northern Colorado, Greeley, CO 80639. tion in order to make an inference. For example, consider a scenario from the lack-of-knowledge condition in our study:

If a child your age knows a lot about fruit but only remembers seeing an apple and orange, and does not remember ever seeing a nectarine, do you think he/she has ever seen a nectarine at some time and just forgets seeing it?

Here the child is given the assertion that a protagonist is an expert about a particular category (fruit), but that the protagonist is only certain about remembering a small number of items in that category. Will these pieces of information provide positive or negative strength in the inference process? Do any of the assertions become "counter factors" in the inference process? When the item that is forgotten is less frequent in a child's encounter (i.e., nectarines are less salient or familiar than, for instance, an apple), how will that information be weighted in the inference process? In short, it is when intellectually gifted and average children are presented with multiple pieces of information that an understanding of the inferential process can occur.

We assume that intellectually gifted and average children understand that a lack of knowledge works to constrain the process of memory. However, we predict that gifted children are more likely to have depressed ratings than are average children when the protagonist lacks memory knowledge. This occurs because gifted children have a uniquely different means of combining internal in- 
formation (e.g., causal schema) and therefore are less susceptible to multiple pieces of external information (i.e., expertise, item familiarity, etc.). The central prediction of this study was that an interaction between ability group and type of inference (knowledge vs. lack of knowledge), expertise (expert vs. novice), salience (high vs. low), and number set (large vs. small) would occur.

\section{METHOD}

\section{Subjects}

The sample $(N=34)$ was randomly selected from children, ages 10 through 15, who attended the 1982 Summer Enrichment Program at the University of Northern Colorado. A random sample of children attending SEP was administered the Otis-Lennon IQ test in a group setting. Subjects were selected whose IQ scores on the Otis-Lennon IQ test were between 100 and 115 or above 135 . The resulting sample consisted of 17 intellectually gifted children (mean IQ $=141.80, S D=$ 3.62; mean $C A=13.42, S D=1.16$ ) and 17 intellectually average (or nongifted) children (mean $I Q=108.23, S D=3.29$; mean $C A=13.35$, $S D=1.05$ ). Fifteen males and 2 females were in each group.

\begin{abstract}
Materials
A set of 32 scenarios, adapted from Gentner and Collins (1981), were constructed that varied with respect to each of the within-group independent variables. Three independent variables used in constructing the scenarios were knowledge of the protagonist, salience of the items, and number of items experienced by the protagonist. Protagonists were depicted as experts (knowing very much) or novices (knowing very little) about the item category in the scenario. Salience of items to be remembered by the protagonist was either high or low (based on norms by Battig \& Montague, 1969). The number of items experienced by the protagonist was either five (larger) or two (smaller). The presentation of the scenarios was in a fixed random order for all subjects with the restriction that replicated versions of a scenario were not adjacent. For each scenario, subjects rated the likelihood that a protagonist would remember an event. The likelihood scale ranged from 1 (impossible) to 5 (certain), with a rating of 3 corresponding to may or may not be possible. Two basic scenarios were prepared, each involving a different type of inference. One type of inference, a memory inference, is a simple induction of whether the protagonist, who has seen certain specific items, would remember seeing another item. An example of one version of scenario shows how the other independent variables entered into the construction. "If a child your age knows little about animals but has seen a dog, cat, horse, cow, and lion, do you think he/she would have remembered seeing a pig?"' (novice, large number set, high-salience condition). The other type of inference, a lack-of-knowledge inference, requires a judgment about whether a protagonist has forgotten about seeing an item, even though that person does remember seeing other categorically related items. The introduction of this article provides one version of a scenario for the lack-of-knowledge condition.
\end{abstract}

\section{Procedure}

Subjects were given the complete set of scenarios in small groups to read and rate individually. All subjects were individually tested. Instructions for rating each scenario were provided in writing and were given verbally by a tester. No restrictions were placed on time for completion, although subjects completed the task in less than 20 min.

\section{RESULTS AND DISCUSSION}

A preliminary analysis of sex difference in likelihood ratings was not significant $(F<1.5)$. Therefore, male and female ratings were not separated in the overall analysis. A five-way analysis of variance performed on likelihood ratings was represented factorially as 2 (groups: gifted vs. average intelligence) $\times 2$ (inference: memory inference vs. lack of knowledge) $\times 2$ (amount of knowledge: expert vs. novice) $\times 2$ (salience: high salience vs. low salience) $\times 2$ (number of items: large set vs. small set) with repeated measures on the last four factors. Table 1 presents the mean likelihood-of-remembering ratings for each group and each condition.

Significant main effects were found for group $[F(1,32)=5.75, p<.05]$, item salience $[F(1,32)=$ $7.08, p<.05]$, and number of items $[F(1,32)=9.50$, $p<.01]$. As shown in Table 1 , children of average intelligence had higher likelihood ratings than did gifted children. For both groups, protagonists were rated more likely to remember if they had experiences with five rather than two items and if the item to be remembered was highly salient. The nonsignificant main effects for type of inference and amount of knowledge $(F \mathrm{~s}<1.0)$ were obscured by a number of interactions. Because the present design was somewhat complex, owing to the number of significant interactions, the analysis was simplified. Only interactions related to ability-group differences were analyzed.

Significant interactions were found for group $\times$ amount of knowledge $[F(1,32)=7.13, p<.05]$, group $\times$ inference $\times$ amount of knowledge $[F(1,32)=4.17$, $p<.05]$, group $\times$ inference $\times$ number of items $[F(1,32)=7.77, p<.01]$, group $\times$ salience $\times$ number of items $[F(1,32)=15.00, p<.01]$, and group $\times$ inference $\times$ amount of knowledge $\times$ salience $\times$ number of items $[F(1,32)=11.02, p<.01]$. Since the number of independent comparisons was high, thus increasing the probability of a spuriously significant result, a post hoc multiple comparison procedure was used. Therefore, the

Table 1

Mean Likelihood Ratings for Gifted and Average Children

\begin{tabular}{|c|c|c|}
\hline & Gifted & Average \\
\hline \multicolumn{3}{|c|}{ Lack-of-Knowledge Inference } \\
\hline \multicolumn{3}{|c|}{ Expert-High Salience } \\
\hline Small Set & 3.30 & 2.90 \\
\hline Large Set & 3.15 & 4.80 \\
\hline \multicolumn{3}{|c|}{ Expert-Low Salience } \\
\hline Small Set & 2.30 & 2.70 \\
\hline Large Set & 2.95 & 3.10 \\
\hline \multicolumn{3}{|c|}{ Novice-High Salience } \\
\hline Small Set & 2.95 & 2.30 \\
\hline Large Set & 3.30 & 3.30 \\
\hline \multicolumn{3}{|c|}{ Novice-Low Salience } \\
\hline Small Set & 3.15 & 3.00 \\
\hline Large Set & 3.00 & 2.90 \\
\hline \multicolumn{3}{|c|}{ Memory Inference } \\
\hline \multicolumn{3}{|c|}{ Expert-High Salience } \\
\hline Small Set & 2.70 & 3.30 \\
\hline Large Set & 3.00 & 3.10 \\
\hline \multicolumn{3}{|c|}{ Expert-Low Salience } \\
\hline Small Set & 3.30 & 3.00 \\
\hline Large Set & 2.95 & 3.10 \\
\hline \multicolumn{3}{|c|}{ Novice-High Salience } \\
\hline Small Set & 3.15 & 3.10 \\
\hline Large Set & 3.45 & 3.70 \\
\hline \multicolumn{3}{|c|}{ Novice-Low Salience } \\
\hline Small Set & 3.00 & 3.50 \\
\hline Large Set & 3.60 & 2.65 \\
\hline
\end{tabular}


interactions vere analyzed via Duncan's Multiple Range Test, and only significant $(p<.05)$ pairwise mean comparisons between groups critical to the predictions of the study are discussed.

As can be determined from Table 1, support is found for the notion that an inference of memory processes takes place when children believe that a protagonist would remember certain experiences. These experiences were more likely to be remembered if the protagonist experienced a large number of items and the information the protagonist was to remember was highly salient. Moreover, differences in children's inference from lack of knowledge are related to intelligence. Children of average intelligence are more likely to rate a protagonist as remembering an event from an incomplete knowledge base than are gifted children. Specifically, children of average intelligence are more likely than gifted children to rate a person who is viewed as an expert, is associated with salient information, and previously remembers a large number set as likely to have the designated item in memory. For the memory-inference condition, children of average intelligence are comparable to gifted children in their memory-inference ratings when the protagonist is a novice but has a knowledge base. However, gifted children are less influenced than are children of average intelligence when the protagonist is an expert, has a large number of experiences, and must retrieve a salient item. The important point is that both gifted and average children's introspective knowledge of memory arises as an interaction between task variables and their existing knowledge of memory processes. Therefore, an intelligent awareness of memory is not an all-or-none phenomenon, but is related to complete or incomplete knowledge. These findings provide some theoretical insights for our understanding of intelligently gifted children. The results support the hypothesis that gifted children have nonstandard or novel ways of sifting out relevant information from irrelevant information, selectively combining relevant pieces of information, and relating new information to previously acquired information (Sternberg \& Davidson, 1983). Thus, these separate but related processes allow for inferences that are not characteristic of normal learners. Although this theory has promise, we are unable in the present experiment to clearly "separate out" the mechanisms responsible for individual differences.

\section{REFERENCES}

Battig, W., \& Montague, W. (1969). Category norms for verbal items in 56 categories: A replication and extension of the Connecticut category norms. Journal of Experimental Psychology Monographs, 80(3, Pt. 2).

Gentner, D., \& Collins, A. (1981). Studies of inference from lack of knowledge. Memory \& Cognition, 9, 434-443.

STERNBERG, R. J. (1982). Teaching scientific thinking to gifted children. Roeper Review, 18, 4-6.

SternberG, R. J., \& Davidson, J. E. (1983). Insight in the gifted. Educational Psychologist, 18, 51-57.

(Manuscript received for publication August 4, 1986.) 